\title{
Uma proposta didático-matemática para o uso da escala de Planck: dos fótons aos buracos negros
}

\author{
A didactic-mathematical proposal for using the Planck scale: from photons to black holes
}

\author{
Caio Matheus Fontinele dos Santos ${ }^{* 1}$, João Marcos Costa da Silva ${ }^{1}$, Érica Cupertino Gomes ${ }^{10}$, \\ Matheus Pereira Lobo ${ }^{1}$ \\ ${ }^{1}$ Universidade Federal do Tocantins, Araguaína, TO, Brasil.
}

Recebido em 18 de dezembro de 2019. Revisado em 14 de maio de 2020. Aceito em 23 de junho de 2020.

\begin{abstract}
Apresentamos, neste artigo, um estudo teórico sobre os limites físicos associados a uma partícula fundamental, o fóton, na escala de Planck. Além disso, calculamos a temperatura de um buraco negro com as dimensões de Planck. A partir da análise dimensional, verificamos cinco unidades da escala de Planck (massa, tempo, comprimento, energia e temperatura). Primeiramente, analisamos um fóton com dimensões do comprimento de Planck; em seguida, identificamos a energia deste fóton com o comprimento de onda da ordem do raio de Schwarzschild $\left(r_{s}\right)$. Por fim, calculamos a temperatura de um buraco negro com as dimensões de Planck (no caso, com seu raio de Schwarzschild igual ao comprimento de Planck). O "raio do fóton" aparece como um múltiplo do comprimento de Planck, demonstrando a inacessibilidade de escalas menores que a de Planck devido ao Princípio da Incerteza. Dentre os resultados obtidos, podemos destacar três caminhos possíveis para a escala de Planck, bem como a mensuração do menor fóton possível de acordo com as leis da Física, que se caracteriza por máxima frequência e energia.
\end{abstract}

Palavras-chave: Fóton, Escala de Planck, Raio de Schwarzschild, Buraco negro.

In this paper we present a theoretical study of the physical limits associated with a fundamental particle, the photon, in the Planck scale. In addition, we calculated the temperature of a black hole with Planck dimensions. From the dimensional analysis, we verified five units of the Planck scale (mass, time, length, energy, and temperature). First, we analyze a photon with dimensions of Planck length; Then we identify the energy of this photon with the wavelength of the order of the Schwarzschild ray $\left(r_{s}\right)$. Finally, we calculate the temperature of a black hole with Planck's dimensions (in this case, with its Schwarzschild radius equal to the Planck's length). The "photon ray" appears as a multiple of Planck's length, demonstrating the inaccessibility of scales smaller than Planck's due to the Uncertainty Principle. Among the results obtained, we can highlight three possible paths to the Planck scale, as well as the smallest possible photon measurement according to the laws of physics, which is characterized by maximum frequency and energy.

Keywords: Photon, Planck scale, Schwarzschild ray, Black hole.

\section{Introdução}

O fóton é a partícula fundamental da natureza que compõe a luz, mediadora da interação eletromagnética, com massa de repouso igual a zero, que se desloca no espaço com a máxima velocidade permitida (a velocidade da luz), segundo a Relatividade Especial. Essa interpretação moderna da luz só foi possível graças aos fenômenos experimentais observados em relação ao comportamento "não clássico" da luz, bem como o comportamento dual (onda e partícula) observado nas partículas elementares massivas. Com o advento da Mecânica Quântica, no início do século XX, houve uma verdadeira revolução na Física com o surgimento de novos conceitos e a alteração de outros preexistentes. A partir de então, percebeu-se

*Endereço de correspondência: caiofontinele22@gmail.com que a luz tem um caráter dual, com comportamentos corpusculares e ondulatórios.

A ideia de tratar a energia de forma "descontínua", ou "discretizada", não foi oriunda do próprio Einstein, mas sim de Max Planck (1858-1947). Planck, um dos pais da Física Quântica, a fim de resolver o problema da radiação de corpo negro (um corpo que absorve qualquer tipo de radiação, não havendo reflexão em relação à radiação incidente), propôs que a energia talvez não fosse transmitida de forma contínua e ininterrupta, como se pensava até então, mas sim em quantidades específicas, em "pacotes" de energia. Planck os nomeou de quanta de energia (cujo singular é quantum), originando a denominação da teoria quântica.

Além de iniciar a Revolução Quântica, Planck, em 1899, encontrou uma forma curiosa de representar as unidades básicas do Sistema Internacional de Unidades (SI), o me- 
tro $[\mathrm{m}]$, o quilograma $[\mathrm{kg}]$ e o segundo $[\mathrm{s}]$. Ele percebeu que as unidades são combinações de três constantes fundamentais da natureza: $c, G$ e $h$, sendo, respectivamente, a velocidade da luz (envolvida nos fenômenos relativísticos), a constante de Gravitação Universal (constante newtoniana) e a constante de Planck (introduzida por ele mesmo com o nascimento da Física Quântica) [1].

Essas três constantes fundamentais estão presentes nas teorias que representam os pilares básicos do universo; são eles, os fenômenos quânticos $(h)$, relativísticos $(c)$ e gravitacionais $(G)$. A velocidade da luz é a constante universal contida no eletromagnetismo de Maxwell (isto é, nas interações eletromagnéticas); assim, trata-se de uma teoria relativística. Claramente, tem-se quatro teorias envolvidas, são elas: a mecânica relativística, a mecânica quântica, o eletromagnetismo, e a gravitação; e duas interações fundamentais, a eletromagnética e a gravitacional.

Apesar das três constantes fundamentais supracitadas serem as mais recorrentes na escala de Planck, outras constantes da natureza também figuram em tal escala, como por exemplo, a constante de Boltzmann, $\left[k_{B}\right]=$ $\left(\frac{m^{2} \cdot k g}{K \cdot s^{2}}\right)$, relacionada a sistemas termodinâmicos, como será apresentado em uma das seções deste trabalho [2].

Essa forma de representar as unidades ficou conhecida como escala de Planck e os significados e interpretações físicas destes valores têm sido buscados desde então. Para termos uma ideia do tamanho ínfimo dessa escala, o ser humano está mais próximo do tamanho do universo do que do comprimento de Planck! Vale ressaltar que o tempo e o comprimento de Planck estão associados às menores quantidades do universo fisicamente realizáveis e, por isso, intimamente relacionados às propriedades da Mecânica Quântica. Por outro lado, as demais grandezas que se encontram na Tabela 1 (massa, energia e temperatura de Planck) caminham na direção oposta, sendo muito grandes quando comparadas com os fenômenos observáveis no âmbito das partículas elementares [1].

\section{Verificação das unidades de Planck}

Primeiramente, vamos verificar cinco unidades básicas da escala de Planck, comprimento, tempo, massa, energia e temperatura. Para isso, precisamos conhecer as unidades das três principais grandezas envolvidas na escala de Planck, $c, G$ e $h$, bem como utilizar a análise dimensional com as unidades dessas três grandezas até encontrarmos as unidades que buscamos para cada uma das medidas supracitadas. Para não sobrecarregar as notações, utilizaremos a seguinte convenção: $[h]=h,[G]=G,[c]=c$. Dessa forma, conhecendo os valores numéricos e suas respectivas unidades, temos:

$$
\begin{gathered}
G=6,67 \times 10^{-11} \mathrm{Nm}^{2} \mathrm{~kg}^{2}\left(\text { ou } \mathrm{m}^{3} / \mathrm{kgs}^{2}\right), \\
c=3,00 \times 10^{8} \mathrm{~m} / \mathrm{s} \\
h=6,60 \times 10^{-34} \mathrm{Js},
\end{gathered}
$$

Tabela 1: Grandezas da escala de Planck.

\begin{tabular}{lcl}
\hline $\begin{array}{l}\text { GRAN- } \\
\text { DEZA }\end{array}$ & EQUAÇÃO & $\begin{array}{l}\text { VALOR NUMÉRI- } \\
\text { CO/ UNIDADE (SI) }\end{array}$ \\
\hline $\begin{array}{l}\text { Comprimento } \\
\text { de Planck } \\
\left(l_{p}\right)\end{array}$ & $\sqrt{\frac{\hbar \cdot G}{c^{3}}}$ & $1,6 \times 10^{-35} \mathrm{~m}$ \\
\hline $\begin{array}{l}\text { Tempo de } \\
\text { Planck }\left(t_{p}\right)\end{array}$ & $\sqrt{\frac{\hbar \cdot G}{c^{5}}}$ & $5,4 \times 10^{-44} \mathrm{~s}$ \\
\hline $\begin{array}{l}\text { Massa de } \\
\text { Planck }\left(M_{p}\right)\end{array}$ & $\sqrt{\frac{\hbar \cdot c}{G}}$ & $2,2 \times 10^{-8} \mathrm{~kg}$ \\
\hline $\begin{array}{l}\text { Energia de } \\
\text { Planck }\left(E_{p}\right)\end{array}$ & $\sqrt{\frac{\hbar \cdot c^{5}}{G}}$ & $1,956 \times 10^{9} \mathrm{~J}$ \\
\hline $\begin{array}{l}\text { Temperatura } \\
\text { de Planck } \\
\left(T_{p}\right) \quad \sqrt{\frac{\hbar \cdot c^{5}}{G \cdot k_{B}^{2}}}\end{array}$ & $1,4 \times 10^{32} \mathrm{~K}$ \\
\hline
\end{tabular}

$$
\hbar=\frac{h}{2 \pi}=1,05 \times 10^{-34} \mathrm{Js}\left(\text { ou } k g \mathrm{~m}^{2} / \mathrm{s}\right) .
$$

As grandezas da escala de Planck utilizadas neste trabalho estão indicadas na Tabela 1 .

A análise dimensional consiste em prever a dependência das grandezas envolvidas em um problema sem, de fato, resolvê-lo. Sua utilidade é vasta, consistindo em uma ferramenta extremamente útil na física, tanto na área teórica quanto na área experimental. Dentre as inúmeras vantagens de se utilizá-la, podemos citar a simplificação de problemas, o aprimoramento da intuição física, e na detecção de erros em cálculos por meio da verificação das unidades físicas envolvidas no problema e da dimensão física do resultado, comparando-a com o esperado para o cálculo 3. Assim, vale a pena destacarmos que uma das bases deste trabalho é apresentar o alcance e aplicabilidade da análise dimensional.

Utilizando a análise dimensional nas constantes em questão, devemos obter uma dimensão de comprimento $(m)$, que será o comprimento de Planck. Assim, inicialmente multiplicamos as unidades de $\hbar$ por G:

$$
\hbar \cdot G=\left(\frac{k g m^{2}}{s}\right) \cdot\left(\frac{m^{3}}{k g s^{2}}\right)=\frac{m^{5}}{s^{3}} .
$$

Após isso, fazendo a razão de (1) por $c^{3}$,

$$
m^{2}=\left[\frac{\left(\frac{m^{5}}{s^{3}}\right)}{c^{3}}\right]=\left[\frac{\left(\frac{m^{5}}{s^{3}}\right)}{\left(\frac{m^{3}}{s^{3}}\right)}\right]=\left(\frac{\hbar \cdot G}{c^{3}}\right),
$$

verifica-se uma medida de comprimento dependente apenas das três constantes fundamentais. Assim, extraindo a raiz quadrada do resultado (2), temos a dimensão de comprimento, representando o comprimento de Planck:

$$
l_{p}=\sqrt{\frac{\hbar \cdot G}{c^{3}}} .
$$

Acredita-se que esse valor seja um limite de comprimento até mesmo para o domínio quântico, e que na escala de Planck, a Relatividade Geral e a Mecânica Quântica 
devem estar de alguma forma unificadas e seus efeitos devem ser comparáveis 4].

Para encontrarmos o tempo de Planck, primeiramente realizamos o mesmo procedimento, multiplicando as unidades de $\hbar$ por $G$, obtendo, assim, o mesmo resultado (1). Em seguida, dividimos (1) por $c^{5}$, resultando em:

$$
s^{2}=\left[\frac{\left(\frac{m^{5}}{s^{3}}\right)}{c^{5}}\right]=\left[\frac{\left(\frac{m^{5}}{s^{3}}\right)}{\left(\frac{m^{5}}{s^{5}}\right)}\right]=\left(\frac{\hbar \cdot G}{c^{5}}\right) .
$$

A partir de (4), extraímos a raiz quadrada para obtermos o tempo de Planck

$$
t_{p}=\sqrt{\frac{\hbar \cdot G}{c^{5}}},
$$

que corresponde ao tempo decorrido para a luz percorrer o comprimento de Planck $\left(l_{p}\right)$.

Para obtermos a massa de Planck, utilizamos a mesma ideia dos cálculos anteriores. Multiplicando as unidades de $\hbar$ por $c$, temos

$$
\hbar \cdot c=\left(\frac{k g m^{2}}{s}\right) \cdot\left(\frac{m}{s}\right)=\left(\frac{k g m^{3}}{s^{2}}\right) .
$$

Dividindo (6) por $G$,

$$
k g^{2}=\left[\frac{\left(\frac{k g m^{3}}{s^{2}}\right)}{\left(\frac{m^{3}}{k g s^{2}}\right)}\right]=\left(\frac{\hbar \cdot c}{G}\right) .
$$

Finalmente, extraímos a raiz quadrada de (7). Assim como nos casos anteriores, obtemos a massa de Planck a partir das três constantes fundamentais,

$$
M_{p}=\sqrt{\frac{\hbar \cdot c}{G}} .
$$

Para obtermos a energia de Planck, precisamos combinar as constantes de forma a encontrar um valor com dimensão de energia, que no Sistema Internacional (S.I.) é dada em Joules $\left([J]=k g \frac{m^{2}}{s^{2}}\right)$. Primeiramente, multiplicamos as unidades de $\hbar$ por $c^{5}$ :

$$
\hbar \cdot c^{5}=\left(\frac{k g m^{2}}{s}\right) \cdot\left(\frac{m^{5}}{s^{5}}\right)=\left(\frac{k g m^{7}}{s^{6}}\right) .
$$

Agora, dividimos (9) pelas unidades de $G$ para obtermos um valor com dimensão de energia:

$$
J^{2}=\left[\frac{\left(\frac{k g m^{7}}{s^{6}}\right)}{\left(\frac{m^{3}}{k g s^{2}}\right)}\right]=\left(\frac{\hbar \cdot c^{5}}{G}\right) .
$$

Dessa forma, extraímos a raiz quadrada do resultado final e obtemos uma grandeza com dimensão de energia, por meio de uma nova combinação das três constantes fundamentais:

$$
E_{p}=\sqrt{\frac{\hbar \cdot c^{5}}{G}}
$$

Por fim, precisamos encontrar a temperatura (em Kelvin), combinando as constantes fundamentais como fizemos anteriormente. Primeiramente combinamos as unidades de $\hbar, c^{5}$ e $G$ da mesma forma que foi realizado em (10),

$$
\left(\frac{\hbar \cdot c^{5}}{G}\right)=\left[\frac{\left(\frac{k g m^{7}}{s^{6}}\right)}{\left(\frac{m^{3}}{k g s^{2}}\right)}\right] \cdot\left(\frac{m^{5}}{s^{5}}\right)=\left(\frac{k g^{2} m^{4}}{s^{4}}\right) .
$$

Em seguida, efetuamos a divisão de 12 por $\left[k_{B}^{2}\right]=$ $\frac{m^{4} K g^{2}}{s^{4} K^{2}}$, sendo $k_{B}=1,38 \times 10^{-23}\left(\frac{m^{2} \cdot k g}{K \cdot s^{2}}\right)$ a constante de Boltzmann. Portanto, temos que a temperatura (em Kelvin) ao quadrado vale:

$$
K^{2}=\left[\frac{\left(\frac{k g m^{7}}{s^{6}}\right)}{\left(\frac{m^{4} K g^{2}}{s^{4} k^{2}}\right)}\right]=\left(\frac{\hbar \cdot c^{5}}{G}\right) \cdot \frac{1}{k_{B}^{2}}=\frac{\hbar \cdot c^{5}}{G \cdot k_{B}^{2}} .
$$

Podemos escrever essa última expressão de uma forma mais fundamental, extraindo a raiz de 13 para, enfim, obtermos um valor com dimensão de temperatura. Combinando, além das três constantes fundamentais como havíamos feito anteriormente, uma nova constante, a constante de Boltzmann,

$$
T_{p}=\sqrt{\frac{\hbar \cdot c^{5}}{G \cdot k_{B}^{2}}} .
$$

Por meio da análise dimensional conseguimos obter as grandezas associadas à escala de Planck, o que evidencia o poder do método. Embora nas últimas décadas esse recurso venha sendo negligenciado devido algumas alterações pedagógicas, chegando a ser eliminada em muitos livros-texto do Ensino Médio e do Ensino Superior [5], não podemos desmerecer suas valiosas contribuições e sua importância para o ensino de Física. Ela desempenha um papel essencial na formação dos estudantes, auxiliando na compreensão das grandezas envolvidas em suas equações e no entendimento da ciência de modo geral [5].

É significativo destacar que a utilidade do método da análise dimensional não se restringe a áreas ou nichos específicos da Física, mas abrange e contempla todas elas. Um exemplo claro é no estudo da Física Moderna, conforme vimos no presente trabalho, bem como em outras áreas, tais como a Química, nos cálculos estequiométricos. É bastante útil na previsão de resultados relevantes sem a resolução direta do problema em si, como no problema do raio de Bohr, estimando o tamanho típico do átomo de hidrogênio ou ponderando a temperatura e a entropia do buraco negro. Mais detalhes e exemplos podem ser encontrados na referência 3 .

\section{O raio de Schwarzschild e o comprimento de Planck}

As constantes descritas pela escala de Planck possuem algumas peculiaridades como serem dimensionalmente 
independentes, ou seja, nenhuma combinação entre elas é adimensional. Cada uma das três constantes está relacionada a uma característica intrínseca da natureza, a constante de Planck $(\hbar)$ indica que a escala de Planck está associada a efeitos quânticos, a velocidade da luz (c) indica que está relacionada ao espaço-tempo e a constante de Gravitação Universal $(G)$ refere-se à gravidade [1].

Embora a confirmação experimental das teorias envolvendo a escala de Planck seja bastante complicada, acredita-se que ela seja um limite para as nossas teorias físicas atuais. Por isso, busca-se interpretações físicas destes valores como, por exemplo, a radiação prevista por Hawking (conhecida como radiação Hawking), que envolve a energia de Planck, onde se acredita que buracos negros podem ter uma temperatura conhecida como "temperatura Hawking". Assim, nos estágios finais da evaporação dos buracos negros, a radiação Hawking emitida por esse buraco negro deverá ter a energia de Planck, fato que ainda não foi observado, mas que os teóricos acreditam ser verdade [1].

O raio de Schwarzschild é uma medida do limite inferior de comprimento que todo corpo material possui. Assim, caso o corpo atinja um comprimento inferior ao de seu raio de Schwarzschild $\left(r_{s}\right)$, haverá o surgimento de uma singularidade, ou seja, o corpo se transformará em um buraco negro 6]. Para tempos menores que $10^{-43} s$ (tempo de Planck), as teorias físicas conhecidas não se aplicam, devido à violação do princípio da incerteza, que proíbe, por exemplo, que duas grandezas, como espaço e momento, tenham simultaneamente valores bem determinados. Aplicaremos o princípio da incerteza para a energia e o tempo a partir da energia de Planck (do nosso universo primitivo) e um tempo menor que o tempo de Planck. Ao fazer isso podemos notar que a desigualdade é violada, pois a incerteza mínima é maior que o produto energia · tempo. Por essa razão, não podemos acessar escalas menores que a de Planck, violando, assim, o princípio da incerteza, já que isso geraria um buraco negro inacessível.

A partir de (3) e (8), temos que

$$
l_{p} M_{p} c=\hbar \text {. }
$$

O princípio da incerteza aplicado a medidas de posição e momento para um fóton com massa (gravitacional) de Planck $\left(p=M_{p} c\right)$ é dado por

$$
(\Delta x) M_{p} c \geq \frac{\hbar}{2}
$$

Assim, para $\Delta x=l_{p}$, temos que as equações (15) e (16) são válidas, isto é, a escala de Planck é compatível com os resultados do princípio da incerteza e da relatividade especial aplicados a um fóton.

Nesta seção, faremos a razão entre o raio de Schwarzschild $\left(r_{s}\right)$ do fóton e o comprimento de Planck, a fim de compararmos esses valores, ou seja, o intuito é encontrarmos quanto o raio limite do fóton representa do comprimento de Planck. O comprimento de onda é a distância pela qual a forma referente a sua frequência começa a se repetir. Nesse sentido, o raio do fóton está relacionado com seu comprimento de onda. Vale destacar que essa é uma extrapolação da relatividade especial para um regime quântico, algo puramente teórico feito aqui com o intuito de testarmos os limites das aplicações de ambas as teorias.

Devemos escrever o raio de Schwarzschild $\left(r_{s}\right)$ do fóton em função das constantes fundamentais $c, G$ e $\hbar$. Para isso, vamos utilizar as seguintes equações para o raio de Schwarzschild $\left(r_{s}\right)$, para a energia do fóton $\left(E_{f}\right)$, para a energia relativística $(E)$ e para a equação da velocidade de uma onda $(c)$ :

$$
\begin{gathered}
r_{s}=\frac{2 G M_{g}}{c^{2}}, \\
E_{f}=\hbar \nu, \\
E=M_{g} c^{2}, \\
c=\lambda \nu .
\end{gathered}
$$

Isolando a massa na equação (19) e substituindo (18) e (20) em (19), obtemos uma expressão para a massa gravitacional do fóton,

$$
M_{g}=\frac{E}{c^{2}}=\frac{\hbar \nu}{c^{2}}=\frac{\left(\frac{\hbar c}{\lambda}\right)}{c^{2}}=\frac{\hbar}{\lambda c} .
$$

Note que o fóton, por ter massa de repouso zero, é uma partícula composta unicamente por energia, por isso utilizamos a equação relativística, $E=m c^{2}$. É importante ressaltar que estamos utilizando um único conceito de massa, uma propriedade intrínseca à partícula, capaz de interagir gravitacionalmente.

Seguimos a abordagem de [7] no sentido de não utilizarmos o conceito de massa relativística, dado que o princípio de covariância de Lorentz garante que a Física (e, portanto, a massa) não é alterada em diferentes referenciais inerciais. Associamos à energia do fóton uma massa gravitacional $M_{g}$. Na equação (21), vemos que a massa gravitacional do fóton é inversamente proporcional ao seu comprimento de onda, isto é, quanto maior seu comprimento de onda, menor sua frequência e, portanto, menos energético ele é.

Substituindo esse resultado na equação do raio de Schwarzschild (17), obtemos uma expressão para o raio desse fóton que depende das constantes fundamentais da escala de Planck,

$$
r_{s}=\frac{2 G M_{e f}}{c^{2}}=\frac{(2 G) \cdot\left(\frac{\hbar}{\lambda c}\right)}{c^{2}}=\frac{2 G \hbar}{\lambda c^{3}} .
$$

Em seguida, substituindo o comprimento de onda $(\lambda)$ em (22) pelo comprimento de Planck, temos:

$$
r_{s}=\frac{2 G \hbar}{l_{p} c^{3}} .
$$

Finalmente, fazendo a razão entre a equação 23 e o comprimento de Planck $\left(l_{p}\right)$ presente na Tabela 1 , temos o seguinte resultado (ilustrado na Figura 1): 


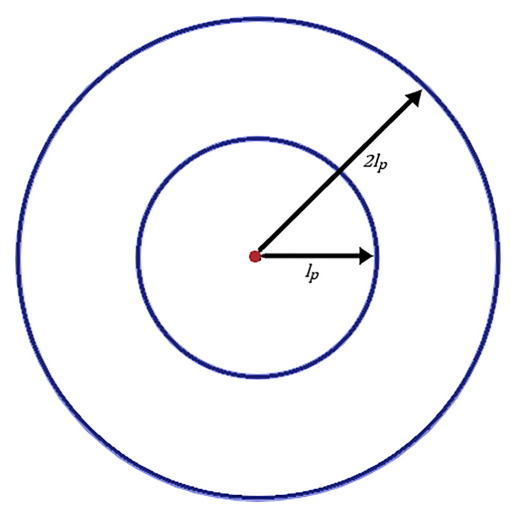

Figura 1: Fóton com raio limite equivalente ao dobro do comprimento de Planck. (Fonte: própria).

$$
\frac{r_{s}}{l_{p}}=\left[\frac{\left(\frac{2 G \hbar}{l_{p} c^{3}}\right)}{l_{p}}\right]=2 \rightarrow r_{s}=2 l_{p} .
$$

Com base no resultado (24), verificamos dois fatos importantes. Primeiro, ao substituirmos o comprimento de onda do fóton pelo comprimento de Planck na expressão (23), obtivemos o menor fóton possível de existir na natureza. Ao fazermos a razão do seu raio limite pelo comprimento de Planck e obtermos o valor 2, verificamos que o raio limite desta partícula é o dobro do comprimento de Planck, como mostra a Figura 1, comprimento este menor que o seu raio de Schwarzschild.

Na escala de Planck, os aspectos quânticos da gravidade se tornam notáveis e o espaço-tempo possivelmente adquire uma estrutura discreta, ou seja, quantizada. Tal fato nos induz à seguinte pergunta: O que é o espaçotempo na escala de Planck? Quando formos capazes de entender essa questão seremos capazes de formular uma teoria quântica da gravidade, fazendo a união da Relatividade Geral com a Mecânica Quântica, um dos feitos mais almejados da Física Contemporânea [8]. Embora já existam muitos trabalhos em relação ao tema, principalmente envolvendo a teoria de cordas e a gravitação quântica em loop, talvez ainda estejamos muito longe de tal realização [4].

\section{A energia do Fóton e a energia de Planck}

Os experimentos atuais na área de Física de Partículas que envolvem altas energias tratam apenas com energias da ordem de $10^{3} \mathrm{GeV}$. Nesse mesmo caminho, podemos citar ainda os raios cósmicos com a mais alta energia detectados até hoje, que possuem uma energia de cerca de $10^{12} \mathrm{GeV}$, o que está MUITO abaixo da energia de Planck, que é da ordem de $10^{19} \mathrm{GeV}[1]$. Isso equivale, em unidades do SI, a $10^{9} \mathrm{~J}$. Dessa análise, podemos notar o quão grande é esta energia.

Nesta seção, partiremos da equação de energia do fóton (18), buscando descobrir sua energia em função das constantes fundamentais da escala de Plank. Primeiramente, substituímos a equação de velocidade da onda (20) na frequência $\nu$ e, em seguida, substituindo o raio de Schwarzschild (17) no comprimento de onda do fóton, como fizemos anteriormente, já que buscamos a energia do menor fóton possível de existir na natureza. Assim, temos:

$$
E=\hbar \nu=\hbar \cdot\left(\frac{c}{\lambda}\right)=\hbar \cdot\left[\frac{c}{\left(\frac{2 G M}{c^{2}}\right)}\right]=\frac{\hbar c^{3}}{2 G M} .
$$

Substituindo $M$ pela equação relativística 19 , temos

$$
E=\left[\frac{\hbar c^{3}}{\left(\frac{2 G E}{c^{2}}\right)}\right]=\frac{\hbar c^{5}}{2 G E}
$$

Isolando o termo de energia, agora presente nos dois membros de 26), obtemos:

$$
E^{2}=\frac{\hbar c^{5}}{2 G} \text { ou } E=\sqrt{\frac{\hbar c^{5}}{2 G}}
$$

Em seguida, fazemos a razão entre essa energia do fóton e a energia de Planck (11):

$$
\frac{E}{E_{p}}=\left(\frac{\sqrt{\frac{\hbar c^{5}}{2 G}}}{\sqrt{\frac{\hbar c^{5}}{G}}}\right)=\sqrt{\frac{\hbar c^{5} G}{2 G \hbar c^{5}}}=\sqrt{\frac{1}{2}}=\frac{\sqrt{2}}{2} .
$$

Dessa forma, obtemos características específicas desse fóton como quando fizemos o comprimento de onda igual ao comprimento de Planck $\left(l_{p}\right)$. Assim, calculamos o fóton com o menor comprimento de onda, ou seja, o menor fóton possível de existir na natureza. Como esse fóton possui o menor comprimento de onda possível, temos também um fóton com máxima frequência que, por meio de sua equação de energia, nos apresenta um fóton com máxima energia.

\section{Temperatura do buraco negro de Planck}

Nesta seção, nosso objetivo é calcular qual a temperatura de um buraco negro com as dimensões da escala de Planck. Os buracos negros são os objetos mais densos do universo, propriedade adquirida ao atingir o raio limite conhecido como raio de Schwarzschild, onde toda sua massa é comprimida em um volume pequeno, deformando o espaço-tempo de tal forma que nada pode escapar ao entrar em seu horizonte de eventos, nem mesmo a luz consegue escapar. Os buracos negros mais simples previstos pela Relatividade Geral são chamados de buracos negros de Schwarzschild, caracterizados por serem estáticos e esféricos 6].

Quanticamente, os buracos negros emitem certo espectro de radiação, revelando que esses objetos astronômicos possuem propriedades termodinâmicas, como temperatura e entropia, fatos esses descobertos por Jacob Bekenstein e Stephen Hawking na segunda metade do século 
XX. É possível descobrir a temperatura desses objetos por meio da equação da referência [4]:

$$
T=\frac{\hbar c^{3}}{8 \pi G k_{B} M_{b}},
$$

onde $M_{b}$ é a massa do buraco negro.

A temperatura de um buraco negro é inversamente proporcional a sua massa $\left(T \propto \frac{1}{M_{b}}\right)$. Isso significa que quanto menor a massa do buraco negro, maior sua temperatura, como é o nosso caso já que a massa de Planck é da ordem de $10^{-8} \mathrm{~kg}$ e a temperatura correspondente é muito alta, da ordem de $10^{31} \mathrm{~K}$. Conforme destacado em [3], "A temperatura de um buraco negro é de natureza quântica, já que se anula quando $h$ é desprezível."

Novamente cabe a ressalva de que estamos utilizando conceitos de relatividade especial e aplicando-os no regime quântico, a fim de podermos concluir sobre os supostos efeitos relativísticos em um regime ultramicroscópio, se tratando, dessa forma, de uma extrapolação teórica. Por meio da equação 29 iremos obter a temperatura de um buraco negro de Planck, isto é, que possua como raio de Schwarzschild o comprimento de Planck. Para isso, iremos utilizar a equação (17), com $r_{s}=2 l_{p}$, colocar a massa em evidência e substituir o raio de Schwarzschild pelo comprimento de Planck $\left(l_{p}\right)$ :

$$
r_{s}=\frac{2 G M_{b}}{c^{2}} ; \quad M_{b}=\frac{c^{2} r_{s}}{G} .
$$

Agora que temos um valor de massa, podemos substituir $M$ de 30 em (29). Assim, obtemos

$$
T=\frac{\hbar c^{3}}{8 \pi G k_{B}\left(\frac{c^{2} l_{p}}{G}\right)}=\frac{\hbar c}{8 \pi k_{B} l_{p}} \sim 10^{31} \mathrm{~K} .
$$

Esse resultado já é conhecido [4]. Como observado na Tabela 1, esse valor é cerca de dez vezes menor do que a temperatura de Planck. Após um período de $10^{-43} \mathrm{~s}$ do Big Bang, essa era a temperatura de toda a matéria que existia até então, que estava em uma rápida expansão acelerada com elevada densidade e uma temperatura colossal [9].

Considerando ordens de grandeza, que no fundo é o que representa nosso entendimento sobre a escala de Planck, as duas energias obtidas na seção anterior, equação (28), são da mesma ordem. O mesmo vale, aproximadamente, para a temperatura de Planck e para a temperatura Hawking do buraco negro na escala de Planck. Esses resultados aproximados, obtidos a partir de diferentes hipóteses e teorias, podem ser justificados por limitações de nosso conhecimento acerca das teorias envolvidas. Essas limitações, por sua vez, correspondem a uma compreensão parcial que temos sobre os fenômenos físicos que estão operando nessa escala. Espera-se que a partir de trabalhos como este, com o intuito de testar nossas melhores teorias em condições extremas, possamos em um futuro breve desvendar o funcionamento do universo em um âmbito mais fundamental.

\section{Considerações Finais}

A escala de Planck pode se tornar uma das áreas mais promissoras da Física se devidamente compreendida, especialmente no que se refere à informação quântica. Atualmente ela representa os processos envolvidos nas mínimas unidades do nosso universo e, portanto, de sua natureza quântica inerente. Suas contribuições permeiam grandes questões científicas ainda não respondidas e fornecem subsídios teóricos para ampliar as teorias já existentes.

A mecânica quântica representa uma das teorias com maior êxito de todos os tempos. As consequências de suas descobertas podem ampliar nosso entendimento acerca das leis naturais bem como contribuir para uma revolução tecnológica de nossa sociedade moderna. A escala de Planck, intimamente relacionada com as partes mais sutis da Mecânica Quântica, pode trazer as respostas para as questões mais intrigantes no momento e abrir uma janela para novas teorias, como já citadas anteriormente, relacionadas ao espaço-tempo e à gravitação quântica [1], e à explicação para a matéria e energia escura na cosmologia. Pode-se conjecturar, por exemplo, que a energia de Planck esteja intimamente relacionada à energia escura 2].

Ela representa uma fronteira para as grandezas físicas e o significado das nossas teorias atuais de gravidade, quanta e espaço-tempo. Por isso não faz sentido, tendo em vista o princípio da incerteza, falarmos em escalas menores que essa.

Os buracos negros estão entre os objetos mais misteriosos do universo, representando grandes desafios teóricos (principalmente no contexto quântico) e experimentais para a comunidade científica. Apesar de se teorizar a existência de corpos com as propriedades dos buracos negros, o advento da Relatividade Geral no início do século XX foi um dos principais responsáveis por fazer os estudos relacionados a esses objetos ter avanços significativos. Por suas características extremas e peculiares, esses corpos são muito importantes, pois podemos testar o limite de nossas teorias físicas. Embora a comprovação direta da existência desses corpos seja problemática, outrora um dos maiores desafios experimentais da física, em virtude de trabalhos recentes, como a detecção das ondas gravitacionais pelo observatório LIGO em setembro de 2015, geradas pela fusão de um sistema binário de buracos negros, bem como as observações de fenômenos semelhantes [10], e pela observação direta do buraco negro da galáxia M87 que se encontra a 53,5 milhões de anos-luz da Terra, foi possível corroborar a teoria e inferir mais evidências concretas da Relatividade Geral 11,13 .

Ressaltamos ainda a utilização de técnicas matemáticas simples, como análise dimensional, com o intuito de tornar este texto didático e compreensível ao público interessado, bem como destacamos a importância da análise dimensional na Física, sendo especialmente útil no entendimento das grandezas físicas e de suas respectivas equações que descrevem a natureza. 


\section{Agradecimentos}

Agradecemos à Universidade Federal do Tocantins por fornecer os subsídios necessários para a produção deste trabalho, bem como ao Mestrado Profissional em Ensino de Física (MNPEF). Destacamos nosso agradecimento ao avaliador pelas valiosas sugestões. Ressaltamos ainda nosso reconhecimento à Sociedade Brasileira de Física (SBF), ao $\mathrm{CNPq}$ pelo apoio financeiro e ao grupo de pesquisa LABID (Laboratório Interativo Digital) pela realização e comprometimento com o trabalho.

\section{Referências}

[1] R.J. Adler, Am. J. Phys 78, 925 (2010).

[2] C. Sivaram, arXiv:0707.0058 (2007).

[3] D. Trancanelli, Rev. Bras. Ens. Fís. 38, e2505 (2016).

[4] V. Faraoni, Am. J. Phys. 85, 865 (2017).

[5] F.W. Silva, Física na Escola 14, 55 (2016).

[6] M.P. Lobo, Physicae 6, 1, (2006).

[7] E.F. Taylor e J.A. Wheeler, Space-Time Physics (W.H. Freeman, San Francisco, 1966).

[8] P.A. Zizzi, arXiv:gr-qc/0304032 (2003).

[9] K.S.O. Filho, Cad. Bras. Ens. Fís 27, 698 (2010).

[10] B.P. Abbott, Phys. Rev. Letters 116, 061102 (2016).

[11] K. Akiyama, A. Alberdi e R. Azualy, Astrophysical J. Letters 875, 17 (2019).

[12] K. Akiyama, A. Alberdi e R. Azualy, Astrophysical J. Letters 875, 28 (2019).

[13] K. Akiyama, A. Alberdi e R. Azualy, Astrophysical J. Letters 875, 32 (2019). 